\title{
Diagnóstico de Orientación Vocacional carrera de Enfermeria primer año. Facultad de Ciencias Médicas "Enrique Cabrera".
}

Diagnosis of Vocational Orientation Nursing career first year. "Enrique Cabrera" School of Medical Sciences.

Diagnóstico de Orientação Vocacional carreira da Enfermeria primeiro ano. Faculdade de Ciências Médicas "Enrique Cabrera".

Ana Iris Santos Romero ${ }^{1}$, Yanelis Perou Silveira ${ }^{2}$, Félix Rene Lima Cabañas ${ }^{3}$, Ayisban Rivera Cabrera ${ }^{4}$, Jacinta Favier Rousseaux ${ }^{5}$.

\section{Resumen:}

Introducción. La orientación vocacional forma parte inevitable del proceso educativo y constituye un requisito imprescindible para que la educación pueda alcanzar su encargo social.

Objetivo. Diagnosticar el nivel de Orientación Vocacional carrera de Enfermeria primer año. Facultad de Ciencias Médicas "Enrique Cabrera”.

Métodos. Se llevó a cabo un estudio descriptivo, de corte transversal en la Facultad de Ciencias Médicas "Enrique Cabrera", Municipio Boyeros, para evaluar el nivel de orientación vocacional de estudiantes en Enfermería primer año, en los cursos escolares 2016-2017 y 2017-2018. La población estuvo constituida por 304 estudiantes. La muestra se obtuvo mediante el muestreo aleatorio simple con 182 estudiantes, a los cuales se les aplicó una encuesta para la recogida de la información con previo consentimiento informado, se realizó análisis documental y estadístico para la obtención, el procesamiento y análisis de los resultados.

\footnotetext{
${ }^{1}$ Licenciada en Enfermería. Asistente. Facultad de Ciencias Médicas Enrique

Cabrera. orcid 0000-0002-2310-924X

e-mail: anairissantosromero119mar@gmail.com

${ }^{2}$ Máster en Longevidad Satisfactoria. Asistente. Facultad de Ciencias Médicas Guantánamo. orcid 00000003-0702-6759X

e-mail: yanelisperousilveira@gmail.com

${ }^{3}$ Máster en Urgencias Médicas. Asistente. Facultad de Ciencias Médicas Salvador Allende. orcid 0000-00033520-0347X

e-mail: rene.limacabanas@gmail.com

${ }^{4}$ Máster en Informática Médica. Asistente. Facultad de Ciencias Médicas Dr. Ernesto Guevara de la Serna. orcid 0000-0002-3160-438X

e-mail: ayisban@gmail.com

${ }^{5}$ Licenciada en Enfermería.Instructor. Facultad de Ciencias Médicas Enrique

Cabrera. orcid 0000-0003-1722-5356

e-mail: jacintafavier@gmail.com
} 
Resultados: El 36,8\% de los estudiantes obtuvo información acerca de la carrera a través de la familia, el 44,4 \% de los estudiantes presentó una deficiente orientación vocacional, el 24,0 \% refirió como motivo principal para elegir la carrera el gusto por ayudar y cuidar, el 46,3\% abandonó la carrera por no vocación.

Conclusiones: Se concluye que la vía fundamental por la que los estudiantes obtienen información acerca de la carrera es a través de la familia, se valora como deficiente la orientación vocacional, se identificó como motivo esencial para elegir la carrera el gusto por ayudar y cuidar, y como causa principal de deserción la no vocación.

\section{Palabras clave:}

Diagnóstico, Orientación vocacional, Educación en Enfermería, Cuba.

\section{Abstract}

Introduction: Vocational guidance is an inevitable part of the educational process and is an essential requirement for education to reach its social order.

Objective: Diagnose the level of Vocational Guidance Nursing career first year. "Enrique Cabrera" School of Medical Sciences.

Method: A descriptive, cross-sectional study was carried out at the "Enrique Cabrera" School of Medical Sciences, Boyeros Municipality, to assess the level of vocational guidance of students in Nursing first year, in the 2016-2017 and 2017-2018 school courses. The population was made up of 304 students. The sample was obtained by simple random sampling with 182 students, to whom a survey was applied for the collection of information with prior informed consent, documentary and statistical analysis was performed to obtain, process and analyze the results.

Results: $36.8 \%$ of the students obtained information about the career through the family, $44.4 \%$ of the students presented a poor vocational orientation, $24.0 \%$ referred as the main reason to choose the career the taste for help and care, $46.3 \%$ abandoned the career because of no vocation.

Conclusions: It is concluded that the fundamental way by which students obtain information about the career is through the family, vocational guidance is valued as deficient, the pleasure to help and care is identified as essential reason to choose the career, and as main cause of defection non-vocation.

\section{Keywords:}

Diagnosis, Vocational guidance, Education Nursing, Cuba. 


\section{Resumo:}

Introdução: A orientação vocacional forma parte inevitável do processo educativo e constitui um requisito imprescindível para que a educação possa alcançar seu encargo social.

Objetivo: Diagnosticar o nível de Orientação Vocacional carreira da Enfermeria primeiro ano. Faculdade de Ciências Médicas "Enrique Cabrera".

Métodos: Levou-se a cabo um estudo descritivo, de corte transversal na Faculdade de Ciências Médicas "Enrique Cabrera", Município Boyeros, para avaliar o nível de orientação vocacional de estudantes em Enfermaria primeiro ano, nos cursos escolar 2016-2017 e 2017-2018. A população esteve constituída por 304 estudantes. Mostra-a se obteve mediante a amostragem aleatória simples com 182 estudantes, aos quais lhes aplicou uma pesquisa para o recolhimento da informação com prévio consentimento informado, realizou-se análise documentário e estatístico para a obtenção, o processamento e análise dos resultados.

Resultados: O 36,8\% dos estudantes obteve informação a respeito da carreira através da família, o 44,4 \% dos estudantes apresentou uma deficiente orientação vocacional, o 24,0 \% referiu como motivo principal para escolher a carreira o gosto por ajudar e cuidar, o 46,3\% abandonou a carreira por não vocação.

Conclusões: conclui-se que a via fundamental pela que os estudantes obtêm informação a respeito da carreira é através da família, valora-se como deficiente a orientação vocacional, identificou-se como motivo essencial para escolher a carreira o gosto por ajudar e cuidar, e como causa principal de deserção a não vocação.

\section{Palavras chave:}

Diagnóstico, Orientação vocacional, Educação em Enfermagem, Cuba.

\section{Introducción}

La orientación vocacional forma parte ineludible del proceso educativo y constituye un requisito imprescindible para que la educación pueda alcanzar su encargo social.

Una adecuada orientación en el campo vocacional desde los primeros años de escolaridad, ayuda al estudiante a seleccionar los estudios más adecuados a sus intereses y competencias. ${ }^{1}$

En la motivación del estudiante para elegir una carrera desempeñan un papel fundamental, las diferentes actividades educativas y sistemáticas de orientación vocacional, desde edades tempranas afín de formar el interés profesional y orientarlo adecuadamente hacia una profesión. ${ }^{2}$ Sin embargo, en la elección de una profesión o trabajo no se pueden dejar de mencionar los factores psicológicos que influyen debido a que tienen que ver con los procesos psíquicos y motivos para elegir una carrera, en el que pueden incidir factores como la familia, y otros. ${ }^{3,4}$

La orientación del estudiante en el mundo de las profesiones depende en parte de las orienta- 
ciones que reciban de sus familiares, maestros y demás. ${ }^{5}$ Pero se consolida a través el proceso educativo, debido a que este se rige por una serie de fundamentos que pueden perfectamente ser aplicados al proceso de orientación vocacional. ${ }^{6}$

De ahí, que la orientación vocacional se considera un trabajo preventivo cuyo propósito fundamental es el de orientar a los estudiantes, para que no realicen una equivocada elección de sus carreras y refuercen sus deseos y motivaciones hacia lo que verdaderamente quieren estudiar y desempeñar., ${ }^{1,7}$

Por tanto, no podemos dejar que la motivación hacia una profesión se desarrolle de manera espontánea sino que se sustente sobre la base objetiva a partir del conocimiento oportuno y adecuado de las disímiles actividades fundamentales de orientación vocacional, que le permita a los estudiantes la posibilidad de conocer los diferentes perfiles laborales en correspondencia con su motivación, actitud y orientación lo cual les permitirá tomar las decisiones de acuerdo con sus capacidades y ubicarse en el contexto socio-laboral. ${ }^{8,9,2}$

En las últimas décadas, una problemática que ha tomado especial relevancia en la formación de recursos humanos en Enfermería es la deserción en los primeros años de la carrera, que en no pocas ocasiones ha sido asociada a diferentes factores entre las que se destacan: el no contar con la adecuada información, dificultades académicas, baja retención escolar, desmotivación, deserción, problemas vocacionales, desinterés por la carrera, entre otros. ${ }^{8}$

Estos elementos demuestran la importancia de que todo estudiante que aspire a ingresar a la carrera de Enfermería, posea la máxima información acerca de en qué consiste la profesión, en todos los posibles ámbitos de desempeño, para que los estudiantes puedan realizar una elección profesional responsable, fundamentada en sus intereses, conocimientos y posibilidades reales y asumir conscientemente su futura profesión.,10 De ahí la necesidad de evaluar los conocimientos precedentes de dicha profesión a través de un diagnóstico, pues constituye un punto de partida para orientar y guiar a los estudiantes de nuevo ingreso, con el fin de disminuir el porcentaje de estudiante que abandona la carrera durante el primer año. Lo antes expuesto motivó a los autores para la realización de un Diagnóstico de Orientación Vocacional carrera de Enfermeria primer año en los cursos 2016 -2017 y 2017-2018.

\section{Metodología}

Se llevó a cabo un estudio descriptivo de corte transversal en la Facultad de Ciencias Médicas "Enrique Cabrera", Municipio Boyeros, para diagnosticar el nivel de orientación vocacional de estudiantes en Enfermería primer año, en los cursos escolares 2016-2017 y 2017-2018. El universo estuvo constituido por 304 estudiantes de primer año de la carrera técnicos en Enfermería, de ellos 204 pertenecen al curso 2016-2017 y 100 pertenecen al curso 2017-2018; la muestra se obtuvo mediante el muestreo aleatorio simple con el $60 \%$ el cual equivale a 182 estudiantes, que quedó conformada de la siguiente forma, 122 estudiantes del curso 2016 - 2017 y 60 estudiantes del curso 2017-2018, a los cuales se les solicitó previamente el consentimiento informado. Como criterios de inclusión se tuvo en cuenta participar en el estudio de manera anónima y voluntaria.

Se aplicaron métodos teóricos, empíricos y estadísticos para la obtención, el procesamiento y el análisis de los datos. Dentro de los métodos teóricos se emplearon: el método análisis-síntesis, 
inducción y deducción para la obtención de un conocimiento más preciso del objeto de estudio.

En el caso de los métodos empíricos se utilizó una encuesta a los estudiantes modificada de Prieto Cordové Y. Universidad de Ciencias Médicas de Camagüey "Carlos J. Finlay y la de SF. Mella Quintero de la Facultad de Estomatología "Villa Clara". La misma fue aplicada de forma grupal en las aulas de dicha institución en presencia de los investigadores en la primera semana de ambos cursos escolares.

Dentro de los métodos estadísticos se empleó la estadística descriptiva la cual permitió procesar los datos para obtener la información a través del cálculo porcentual. Para la recogida de la información general se elaboró una encuesta tomando como referente las utilizadas en estudios anteriores y que fue previamente validada por aplicación de un plan piloto. Las variables a evaluar en dicha encuesta en ambos cursos escolares fueron: 1) vías por la que los estudiantes obtienen información acerca de la carrera de Enfermería. 2) nivel de orientación.3) motivos para elegir la carrera, 4) causas de deserción. Además, se utilizó el informe final de promoción de los cursos 2016-2017 - 2017 - 2018, para obtener los datos en relación con el comportamiento de la retención escolar, se analizó el registro de matrícula y graduados, y determinar las causas de deserción escolar en ambos cursos estudiado.

El estudio se desarrolló en dos cursos: obtención y comparación de los datos del curso 2016-2017 y 2017-2018 teniendo en cuenta la similitud de los mismos. En la primera etapa, se les solicitó el consentimiento informado a los estudiantes de forma previa, y se tuvieron en cuenta las consideraciones éticas que exigen las investigaciones en humanos. Se elaboró un registro con las variables que midieron el nivel de orientación vocacional perteneciente al curso 2016-2018. Se analizaron los datos obtenidos de la encuesta del curso 2016-2017, después se añadieron los datos obtenidos del informe final de promoción. De igual modo se registraron y analizaron los datos del curso 2017-2018. En la segunda etapa se confrontaron los datos obtenidos de ambos cursos escolares para diagnosticar el nivel de orientación vocacional en el período estudiado.

La información resumida en el registro se procesó de manera automatizada para lo cual se estableció una base de datos. Los resultados fueron ordenados en tablas estadísticas, que tuvieron frecuencias absolutas y porcentajes, se tuvo en cuenta la valoración de los indicadores que midieron el nivel de orientación vocacional y la deserción, además de la información recibida acerca de la profesión y la relación entre la motivación por la carrera y su selección en los cursos 2016-2017 y 2017-2018.comparándo ambos cursos estudiado y con otros afines.

\section{Discusión}

En relación a los resultados se puede concluir que la familia, es fuente importante de influencia en el proceso de ayuda, en cuanto a la valoración acerca de la profesión a elegir ${ }^{8}$ debido a que la mayoría de los estudiantes sólo recibieron información a través de ella. Se coincide, con un estudio realizado por Valdés Carrillo en la facultad de Ciencias Médicas "General Calixto García”, en el curso 2013-2014, en la que declara en su investigación que la mayoría de los estudiantes solo recibieron información a través de la familia. ${ }^{8}$ Es interesante destacar la importancia que se le concede a la familia en la vida del ado- 
lescente, pues es precisamente en el hogar donde se construyen las bases, sobre las cuales el estudiante puede identificar sus aptitudes para la elección de su futura profesión, debido a que esta influye en la decisión profesional de sus hijos cuando expresa sus experiencias, opiniones y otros, ${ }^{5}$ de ahí la importancia de orientar a la familia para que acompañen a sus hijos en proceso de orientación vocacional, púes la elección profesional es una de las tareas de mayor relevancia dentro del proyecto de vida, decisión que se toma a temprana edad y muchas veces sin el adecuado nivel de conocimiento y madurez. ${ }^{11}$ Por tanto, surge la necesidad de desarrollar un trabajo de conjunto escuela, familia, el cual debe estar encaminado a orientar a la misma con el fin de aportarle los conocimientos necesario y preparación para que apoyen a sus hijos en el proceso de elección profesional. ${ }^{12}$

En cuanto a la valoración acerca del nivel de orientación vocacional para la carrera de Enfermerìa, la mayoría de los estudiantes la valoró de mal, lo que reflejó el insuficiente conocimiento sobre la carrera a través de las diferentes vías curriculares o extracurriculares. Se coincide con una investigación realizada por Aúcar López ${ }^{9}$ en la que declara en su investigación el insuficiente conocimientos que poseen los estudiantes sobre la profesión de Estomatología y Prótesis Estomatológica, de igual manera en un estudio realizado por Valdés Carrillo ${ }^{8}$ enuncia que los estudiantes ingresan a la Universidad sin tener los conocimientos necesarios en relación con lo que desean estudiar debido a que se constata la falta de información en cuanto a la profesión de Enfermería en estudiantes de nuevo ingreso. Estas valoraciones demuestran la importancia de que al margen de la inclinación vocacional que puedan sentir los estudiantes hacia la carrera de Enfermería y otras profesiones, es necesario el conocimiento oportuno y adecuado de, sus programas de formación, escenarios donde se desarrolla, perfil del egreso, sus perspectivas en el mercado laboral, las actividades que realiza, objetivo principal de dicha profesión, horarios de trabajo, vías de superación y otros. Todos estos son aspectos decisivos que los ayudan a analizar sobre su futuro profesional, además de que les permita ratificar o no la selección de la profesión. ${ }^{10,9}$ De ahí que la orientación vocacional es una condición indispensable para el éxito de la actividad profesional debido a que es la existencia de una orientación motivacional fundamentada en sólidos intereses profesionales lo que permite un desempeño con calidad y eficiencia, al mismo tiempo que posibilita la plena satisfacción del hombre en su ejercicio. ${ }^{10}$ Por ende se debe continuar perfeccionando el trabajo de orientación vocacional, de manera tal que ayude en el ofrecimiento de valoración a cerca de la profesión a seleccionar, para que los jóvenes seleccionen cada vez mejor sus estudios de acuerdo con sus aptitudes e intereses personales ,sociales y motivaciones. ${ }^{7}$

Es evidente, que la motivación constituye la piedra angular para una correcta elección profesional por ser la fuerza o el incentivo que conduce a la acción ' sin embargo la motivación también puede ser: debido a motivación intrínseca o extrínseca, lo primero proviene del interior, ${ }^{3,4}$ a través, de una orientación vocacional desarrollada en los estudiantes desde edades temprana con una adecuada apropiación de conocimientos, habilidades, valores donde se relacione lo instructivo y lo educativo para esclarecer lo que realmente se desea estudiar' lo 
segundo proviene del exterior' de los cuales se experimenta el control externo en el que pueden incidir la familia, los grupos y otros factores circunstanciales. ${ }^{3,4}$ Por tanto, independientemente de la labor que desarrolla una persona, debe existir un motivo para su realización la motivación puede ser definida como la actitud que presentan las personas al realizar una actividad, la cual permite direccionar su comportamiento, a través de un proceso dinámico interno que puede dar respuesta a la variabilidad en el comportamiento humano. ${ }^{13}$

En relación a las causas de deserción escolar, como unos de los factores que influyeron en el abandono de estudio y por consiguiente en la retención escolar se coincide con una investigación realizada por. Blanca N Piratoba $\mathrm{H}$. en la cual plantea que la deserción escolar constituye uno de los principales problemas en la formación de recursos humanos en Enfermería, debido a que al factor vocacional es uno de los factores más frecuentes para el abandono de la carrera ${ }^{14}$. Estudios realizados en Costa rica, Chile, Paraguay, Colombia entre otros países, incluyendo Cuba declaran entre los motivos de mayor consideración, en cuanto a deserción, la falta de preparación con la que llegan los estudiantes a la universidad, los problemas académicos y vocacionales entre otros. ${ }^{14}$ por tanto para alcanzar mayoresy mejores resultados en la orientación vocacional es necesario que los estudiantes valoren adecuadamente sus posibilidades reales atendiendo a su trascendencia académicos y aptitudes para la selección profesional ya que en ocasiones sus intereses vocacionales no se corresponden a sus posibilidades reales, lo que con lleva en mucho casos al abandono de estudio falta de interés. ${ }^{8}$ De ahí que, la descripción de los planes de estudio des- empeña un papel fundamental en la orientación vocacional de los estudiantes, por permitir elegir su profesión u ocupación atendiendo a su rendimiento académico, elemento esencial a la hora de elegir una profesión u ocupación. ${ }^{9}$ Otro aspecto a señalar es el trabajo con la familia ya que en ocasiones las expectativas de los padres no se corresponden con las capacidades reales de sus hijos' por tanto, la familia debe tener conocimiento de las dificultades académicas para que puedan ayudarlo en la adecuada orientación vocacional y elegir satisfactoriamente su profesión, en el que se deben desarrollar diferentes acciones con el estudiantado, padre, madre, personal encargado y docentes, con el fin de favorecer el desarrollo integral del estudiante y de esta manera concluir exitosamente la carrera más a fin sus intereses, aptitudes y realidades. ${ }^{12}$

\section{Conclusiones}

El diagnóstico realizado permitió valorar que la vía fundamental por la que los estudiantes obtienen información acerca de la carrera de Enfermería es a través de la familia y no de profesionales capacitado para esa actividad. La orientación vocacional en los grupos estudiados se calificó de deficiente, las instituciones en la educación precedente no desempeñaron el rol que les corresponde. A pesar de las deficiencias planteadas, en los estudiantes existió un grupo de motivos que jugaron un papel jerárquico en la toma de decisiones, los motivos fundamentales para la elección de la carrera referido por los estudiantes fue el gusto por ayudar y cuidar lo que destaca su actitud altruista. La principal causa de deserción escolar fue la vocación seguida del bajo rendimiento académico, lo que se evidenció en el comportamiento de la retención escolar. 


\section{Tablas Resultados}

Tabla.1 Vías por la que los estudiantes obtienen información acerca de la carrera de Enfermería curso. 2016-2017- 2017-2018.

\begin{tabular}{|c|c|c|c|c|c|c|}
\hline \multirow{2}{*}{$\begin{array}{l}\text { Vías por la que los estudiantes obtienen } \\
\text { información acerca la carrera de } \\
\text { Enfermería }\end{array}$} & \multicolumn{2}{|c|}{ Curso.2016.2017 } & \multicolumn{2}{|c|}{ Curso.2017.2018 } & \multirow[t]{2}{*}{ Total } & \multirow[t]{2}{*}{$\%$} \\
\hline & Cantidad & $\%$ & Cantidad & $\%$ & & \\
\hline Familia & 70 & 34,3 & 42 & 42 & 112 & 36,8 \\
\hline Comunidad & 6 & 2,9 & 5 & 5 & 11 & 3,6 \\
\hline Escuela & 8 & 3,9 & 6 & 6 & 14 & 4,6 \\
\hline Medio de difusión & 10 & 4,9 & 1 & 1 & 11 & 3,6 \\
\hline Amistades & 13 & 6,3 & 2 & 2 & 15 & 4,9 \\
\hline $\begin{array}{l}\text { Puertas abiertas en la Universidad } \\
\text { ciencias Médicas }\end{array}$ & 15 & 7,3 & 4 & 4 & 19 & 6,2 \\
\hline Total & 122 & 100 & 60 & 100 & 182 & 100 \\
\hline
\end{tabular}

Fuente: Encuesta

Tabla.2 Valoración del nivel de orientación vocacional para la carrera de Enfermería curso.

2016 -2017-2017-2018.

\begin{tabular}{lccccccc}
\hline Nivel de orientación vocacional & \multicolumn{2}{c}{ Curso 2016-2017 } & \multicolumn{2}{c}{ Curso.2017-2018 } & \multicolumn{2}{c}{} \\
& Cantidad & $\%$ & Cantidad & $\%$ & Total & $\%$ \\
\hline Buena & 12 & 5,8 & 6 & 6 & 18 & 5,9 \\
Regular & 19 & 9,3 & 10 & 10 & 29 & 9,5 \\
Mala & 91 & 44,6 & 44 & 44 & 135 & 44,4 \\
\hline Total & 122 & 100 & 60 & 100 & 182 & 100 \\
\hline
\end{tabular}

Fuente: Encuesta

Tabla.3 Motivos para elegir la carrera de Enfermería curso 2016-2017 - 2017 -2018.

\begin{tabular}{lcccccc}
\hline Motivos para elegir la carrera de Enfermerìa & \multicolumn{2}{c}{ Curso.2016.2017 } & \multicolumn{2}{c}{ Curso.2017.2018 } & & \\
& Cantidad & $\%$ & Cantidad & $\%$ & Total & $\%$ \\
\hline El gusto por ayudar y cuidar & 52 & 25,4 & 22 & 22 & 74 & 24,3 \\
Reconocimiento social de la profesión & 12 & 5,8 & 11 & 11 & 23 & 7,5 \\
Para acceder a otra carrera de la salud & 11 & 5,3 & 7 & 7 & 18 & 5,9 \\
Influencia familiar & 10 & 4,9 & 5 & 5 & 15 & 4,9 \\
Siempre quiso ser Enfermera/o & 9 & 4,4 & 5 & 5 & 14 & 4,6 \\
Influencia de amigos & 10 & 4,9 & 4 & 4 & 14 & 4,6 \\
Conocimiento previo de la labor de Enfermería & 8 & 3,9 & 2 & 2 & 10 & 3,2 \\
Uso de uniforme & 6 & 2,9 & 2 & 2 & 8 & 2,6 \\
Orientaciones recibida en la escuela & 4 & 1,9 & 2 & 2 & 6 & 1,9 \\
\hline Total & 122 & 100 & 60 & 100 & 182 & 100 \\
\hline
\end{tabular}

Fuente: Encuesta 
Tabla.4 Causas de deserción escolar en estudiantes de Enfermería primer año curso 2016-2017-2017-2018.

\begin{tabular}{llllllll}
\hline $\begin{array}{l}\text { Causas de deserción escolar } \\
\text { estudiantes de Enfermerìa primer año }\end{array}$ & en & \multicolumn{2}{c}{ Curso2016-2017 } & \multicolumn{2}{c}{ Curso 2017-2018 } \\
Cantidad & $\%$ & Cantidad & $\%$ & Total & $\%$ \\
\hline No vocación & 123 & 60,2 & 18 & 18 & 141 & 46,3 \\
Problemas académicos & 24 & 11,7 & 8 & 8 & 32 & 10,5 \\
Problemas de salud & 1 & 0,4 & 1 & 1 & 2 & 0,6 \\
Embarazo & 2 & 0,9 & 1 & 1 & 3 & 0,9 \\
\hline Total & 150 & 73,5 & 28 & 28 & 178 & 100 \\
\hline
\end{tabular}

Fuente: Registro de matrícula y graduado

Tabla.5 Comportamiento de la retención escolar en estudiantes de primer año carrera Técnicos en Enfermería curso 2016-2017 - 2017 - 2018

\begin{tabular}{lcccc}
\hline Curso & Matrícula inicial & Matrícula final & Bajas & \% de retención \\
\hline $2016-2017$ & 204 & 54 & 150 & 73,5 \\
$2017-2018$ & 100 & 72 & 28 & 28 \\
\hline
\end{tabular}

Fuente: Acta final de promoción en la enseñanza técnica

Referencias Bibliográficas:

1-Prieto Cordovés Y, Guillemí Álvarez N M, Claro Toledo Y. Caracterización de la orientación vocacional en estudiantes de primer año de Medicina. Rev Hum Med [Internet].2019 Ago [citado 04feb2020];19(2):356371Disponibleen:http:// scielo.sld.cu/scielo.php?script=sci_abstract\&pid $=$ S172781202019000200356\&ln$\mathrm{g}=\mathrm{es} \& \mathrm{nrm}=\mathrm{iso} \& \mathrm{t} \operatorname{lng}=\mathrm{es}$

2-Amechazurra Oliva MA, Santana Hernández M, Álvarez Dueñas O. Orientación profesional de los estudiantes: un reto para los docentes de las ciencias médicas. Edumecentro [Internet].20 17Jun[citado 05abr2018];9(2):17998. Disponibleen:http://scielo.sld.cu/pdf/edu/ v9n2/edu13217.pdf
3- Salabert Tortoló I, Naipe Delgado MC, Garriga Alfonso NE, Alfonso Prínce JC, Dihigo Faz MT, Núñez Valdés L. La motivación profesional en los estudiantes de las Ciencias Médicas. Rev Med Electrón [Internet]. 2017 Jun [citado 05 enr 2018]; 39(3):630-9. Disponible en: http:// scielo.sld.cu/scielo.php?script=sci_arttext \&pid=S1684-18242017000300023

4- Naipe Delgado MC, Salabert Tortoló I, Morales Díaz M, Mestre Cárdenas VA, Garriga Alfonso NE, Toledo Martínez TE. La motivación en los estudiantes de primer año de la carrera de Medicina. Curso 2015-2016. Rev Med Electrón [Internet]. 2017Ago [citado 05 ene 2018]; 39(4):906- 
15. Disponible en: http://scielo.sld.cu/pdf/ rme/v39n4/rme060417.pdf

5- García Elizalde A. Orientación profesional responsable. GRANMA. [Internet]. 2018; sección cuba.col.1 [citado 26 mar 2019]. Disponible en: http:/www.granma.cu/ cuba/2018-03-27/orientacion-profesional-responsable-27-03-2018-21-03-06

6- Barbón Pérez OG, Estrada García J, Fonseca Morales RT, Cadena Figueroa MN, Urda Melón O. Modelo de escala pedagógica en el desarrollo de las intenciones profesionales para las carreras de la salud. Revista Cubana de Reumatología [Internet]. 2017Ago[citado04 ene 2018 ]; 19(2):91-8. Disponible en: https://www.redalyc.org/ pdf/4516/451652100006.pdf

7- Contreras Pérez JM, Mirabal Díaz JM, Fong Zurbano GL, Machado Contreras MD, de la Hoz Rojas L, Cobo Lueje MR. Visita virtual a la Facultad de Estomatología: pertinencia en la orientación profesional de la carrera. Edumecentro [Internet]. 2017sep [citado 05 ene 2018]; 9 (3):232-48. Disponible en: http://scielo.sld.cu/pdf/edu/ v9n3/edu15317.pdf

8- Valdés Carrillo AG, Valdés Carrillo ÁM, Fernández Oliva B. Estrategia pedagógica para elevar la formación vocacional y orientación profesional de los estudiantes de Enfermería en la Facultad "Gral. Calixto García", 2013-2014. Educ Med Super [Internet]. 2016Jun [citado 05 mar 2018]; 30(2). Disponible en: http://scielo.sld.cu/scielo.php?script=sci_arttext\&pid=S0864-21412016000200004

9- Aúcar López J, Castañeda Casal L, Landrián Díaz CI, Lajes Ugarte M. Proyec- to comunitario infantil de formación vocacional con participación de estudiantes de Estomatología. Edumecentro [Internet].2017Jun[citado04ene2018];9(2):6375. Disponibleen:http: / / scielo.sld. $\mathrm{cu} / \mathrm{scielo}$.php?script=sci_arttext\&pid=S2077-28742017000200006

10- Díaz Izaguirre Y, Cruz Cruz CL, Suárez Garay $\mathrm{A}$. Reafirmación profesional en los estudiantes: desafío para los docentes de la educación superior. Edumecentro [Internet]. 2019 Sep[citado13ene2020];11(3):4560. Disponibleen: http: / / scielo.sld. $\mathrm{cu} / \mathrm{scielo}$.php? script=sci_arttext\&pid=S2077-28742019000300045

11-Alfaro-Barquero A, Chinchilla-Brenes S. Construcción y validación de un instrumento de evaluación de preferencias y habilidades vocacionales para carreras científico-tecnológicas. Tecnología en Marcha [Internet]. 2017Dic [citado25 feb 2019]; 30(4):138-49. Disponible en: https://www. scielo.sa.cr/pdf/tem/v30n4/0379-3982tem-30-04-138.pdf

12-Carvajal Hernández BM, Castro Tapia OC, Aguilera Gómez RB. Análisis histórico de la orientación a la familia en niños de la infancia preescolar, en cuba. Conrado [Internet]. 2018 Jun [citado15mar 2019marzo15];14(62):328.Disponibleen:http:// scielo.sld.cu/scielo.php?script=sci_arttext\&pid=S1990-86442018000200005

13- Herrera Moya VA, Alfonso Tejeda I, Quintana Uguando M, Pérez Ineránty M, Navarro Aguirre L, Sosa Fleites IM. Formación vocacional y motivación: su incidencia en el estudio de la carrera de Medicina. Edumecentro [Internet]. 2018 Jun [citado 23 ene 
2019]; 10(2):111-25. Disponibleen:http:// scielo.sld.cu/scielo.php?script=sci_arttext\&pid=S2077-28742018000200009

14- Piratoba HBN, Oviedo O, Barbosa ChOO. Factores de deserción de los estudiantes en la facultad de enfermería de la universidad de ciencias aplicadas y ambientales U.D.C.A, durante el periodo: 2009-2010-i 2011. Rev UDCA Actualidad y Divulgación Científica [Internet]. $2013 \mathrm{Dic}[\mathrm{Ci}$ tado27ene2016];16 (2):55362Disponibleen:http://www.scielo.org.co/pdf/rudca/ v16n2/v16n2a31.pdf

15- Prieto Cordovés Y, Labrada Salvat C, Hernández Cuan C, Quesada Molina D. Ca- racterización de la orientación vocacional en estudiantes del ciclo básico de la carrera de Medicina. Educ Med Super [Internet].2012Jun [citado 2019 Sep 02]; 26(2):244-51. Disponible en: http://scielo. sld.cu/scielo.php?script=sci_arttext \&pi$\mathrm{d}=$ S0864-21412012000200007

16- de la Mella Quintero SF, Mirabal Díaz JM, Contreras Pérez JM. Factores motivacionales que influyeron en estudiantes de primer año para elegir la carrera de Estomatología. Edumecentro[Internet].2012; [Citado06mar 2015]4(3):18897.Disponibleen:http://scielo.sld.cu/scielo.php?script=sci_arttext\&pid=S2077-28742012000300019 